\title{
Market frictions and corporate finance: An overview paper
}

\author{
Rochet, Jean-Charles ; Moreno-Bromberg, Santiago
}

\begin{abstract}
We present an overview of corporate-finance models where firms are subject to exogenous market frictions. These models, albeit quite simple, yield reasonable predictions regarding financing, pay-outs and default, as well as asset-pricing implications. The price to pay for the said simplicity is the need to use non-standard mathematical techniques, namely Singular and Impulse Stochastic Control. We explore the cases where a firm with fixed expected profitability has access to costly equity issuance as a refinancing possibility, and that where issuance is infinitely costly. We also present a model of bank leverage.
\end{abstract}

DOI: https://doi.org/10.1007/s11579-014-0121-5

Posted at the Zurich Open Repository and Archive, University of Zurich ZORA URL: https://doi.org/10.5167/uzh-99485

Journal Article

Published Version

Originally published at:

Rochet, Jean-Charles; Moreno-Bromberg, Santiago (2014). Market frictions and corporate finance: An overview paper. Mathematics and Financial Economics, 8(4):355-381.

DOI: https://doi.org/10.1007/s11579-014-0121-5 


\title{
Market frictions and corporate finance: an overview paper
}

\author{
Santiago Moreno-Bromberg · Jean-Charles Rochet
}

Received: 5 August 2014 / Accepted: 21 August 2014 / Published online: 7 September 2014

(C) Springer-Verlag Berlin Heidelberg 2014

\begin{abstract}
We present an overview of corporate-finance models where firms are subject to exogenous market frictions. These models, albeit quite simple, yield reasonable predictions regarding financing, pay-outs and default, as well as asset-pricing implications. The price to pay for the said simplicity is the need to use non-standard mathematical techniques, namely singular and impulse stochastic control. We explore the cases where a firm with fixed expected profitability has access to costly equity issuance as a refinancing possibility, and that where issuance is infinitely costly. We also present a model of bank leverage.
\end{abstract}

Keywords Leverage - Liquidity management - Equity issuance - Singular stochastic control $\cdot$ Stochastic impulse control

Mathematical subject classigications $91 \mathrm{G} 10 \cdot 91 \mathrm{G} 50 \cdot 91 \mathrm{G} 80$

\section{Introduction}

Our objective in this paper is to provide the reader with an overview of the techniques and results of certain continuous-time, corporate-finance models that study how exogenous market frictions (such as the lack of access to bank credit and financial markets, or securityissuance costs) influence corporate decisions regarding financing, pay-outs and default. The modeling philosophy is the following: Consider a firm whose net earnings may be negative, which is captured by the assumption that cashflows follow an arithmetic Brownian motion.

In honor of Ivar Ekeland's seventieth birthday.

S. Moreno-Bromberg $(\varangle) \cdot$ J.-C. Rochet

Institut für Banking und Finance, Universität Zürich, Plattenstr. 32, 8032 Zürich, Switzerland

e-mail: santiago.moreno@bf.uzh.ch

J.-C. Rochet

Toulouse School of Economics (IDEI), Toulouse, France

e-mail: jean-charles.rochet@bf.uzh.ch 
As a consequence, it is possible that the firm runs out of cash and is therefore unable to fund its day-to-day operations. At such point the firm defaults unless it is refinanced. By contrast, when cash reserves are high enough the firm's manager may decide to distribute dividends to the shareholders. What this target cash policy level should be and how it depends on the firm's characteristics is one of our main concerns. We first present in Sect. 2 the results of Jeanblanc and Shiryaev [12] and Radner and Shepp [20], where the manager only decides on the dividends, but refinancing is infinitely costly. Next, in Sect. 3 we extend the model to allow for costly equity issuance. This is a simplified version of the model studied by Décamps et al. in [6]. Finally, in Sect. 4 we present the results of Rochet and Villeneuve [21], who adapt the model in [12] to study a Merton-style problem where portfolio choice is not made by an individual investor, but by a bank. Further readings are suggested in Sect. 5. All proofs are presented the Appendix.

\section{Infinite frictions}

We consider a firm, held by a diffuse base of risk-neutral shareholders, that has fixed expected profitability and that holds no debt. ${ }^{1}$ The shareholders discount the future at the strictly positive rate $r$. The firm generates random net earnings that follow the diffusion process

$$
d Y(t)=\mu d t+\sigma d W(t), \quad Y(0)=y,
$$

where $\mu, y$ and $\sigma$ are positive and $\{W(t), t \geq 0\}$ is a standard Brownian motion defined on the probability space $(\Omega, \mathcal{A}, \mathbb{P})$ and generating the filtration $\mathcal{F}=\left(\mathcal{F}_{t}, t \geq 0\right)$. Given that $\mu$ is greater than zero, the firm is profitable. It may, however, incur in operating losses, since $\sigma$ is positive. Without the possibility of refinancing via equity of debt issuance, the firm must hold a positive amount of cash to finance its operations. The manager must then decide on when to distribute dividends so as to optimally manage the firm's cash reserves.

\subsection{Cash management and dividend policy}

Managerial decisions concerning dividend distribution take place continuously. The only restriction we impose on dividends is that they should be non-negative. ${ }^{2}$ For technical reasons, it is simpler to work with the cumulative dividends, which are then non-decreasing. Formally, a CUMULATIVE DIVIDEND PROCESS is any non-decreasing, $\mathcal{F}$-adapted and càdlàg process $L=\{L(t), t \geq 0\}$, i.e. $L(t)$ represents the total, non-discounted dividends paid out over $[0, t]$. The set of all admissible dividend policies will be denoted by $\mathscr{A}$.

We assume for the time being that the firm has no access to financial markets (or that new equity issuance is infinitely costly) or bank credit. If the manager chooses the dividend policy $L \in \mathscr{A}$, the dynamics of the firm's cash reserves are described by the stochastic differential equation

$$
d R^{L}(t)=d Y(t)-d L(t), \quad R^{L}(0)=x .
$$

In other words, the firm's cash reserves increase (or decrease) via its net earnings and decrease whenever dividend distribution takes place. No interest is earned on cash reserves. ${ }^{3}$ It need not be the case that $y=x$ : We show below that for large-enough levels of $x$, there might be

\footnotetext{
1 Debt financing is sub-optimal in this model, since we consider neither taxes nor moral-hazard problems

2 Negative dividends are equivalent to new equity issuances. They are studied in Sect. 3.

3 This assumption may be relaxed, as done by Décamps et al. in [6].
} 
an exceptional lump-sum dividend paid out to the shareholders at $t=0$. We stress that, for reasons of limited-liability, reserves may only be increased via retained earnings and not by infusions of outside capital.

To meet operating costs the firm's cash reserves must remain non-negative. The firm is liquidated at zero value ${ }^{4}$ whenever $R^{L}(t)=0$. Notice that dividend distribution carries an implicit cost, given that it increases the risk of the reserve levels becoming non-positive. The BANKRUPTCY TIME $\tau_{L}$ associated to a dividend-distribution strategy $L \in \mathscr{A}$ is defined as

$$
\tau_{L}:=\inf \left\{t>0 \mid R^{L}(t) \leq 0\right\}
$$

The manager must balance the trade-off between accumulating reserves, which is inefficient due to discounting, and paying out dividends, which increases the risk of bankruptcy. As a consequence of the structure of the net cashflows, even if the manager were to choose $L \equiv 0$, there would always be a positive probability that the firm would go bankrupt. This issue is exacerbated when any other $L \in \mathscr{A}$ is chosen.

Proposition 2.1 If $0<s<\tau_{L}$ then it holds that $\mathbb{P}\left\{\tau_{L}<t \mid R^{L}(s)\right\}>0$ for all $t>s$.

\subsection{The value function and the first-best}

The manager's objective is to maximize shareholder value. ${ }^{5}$ The latter is defined as the expected, discounted stream of dividends paid out during the firm's lifetime. For a given choice of $L$, shareholder value is represented by the quantity

$$
V^{L}(x):=\mathbb{E}_{x}\left[\int_{0}^{\tau_{L}} e^{-r t} d L(t)\right] .
$$

The operator $\mathbb{E}_{x}[\cdot]$ is the expectation conditional on $R^{L}(0)=x$. An important observation is that the model is stationary, since the cash reserves have a Markovian structure and there is no pre-specified time horizon. This implies that, at any date, the current level of cash reserves, which in the sequel is also denoted by $x$, plays the role of the sole STATE VARIABLE. We follow a dynamic-programming approach and analyze the problem through the properties of the value function

$$
V(x):=\sup _{L \in \mathscr{A}} V^{L}(x),
$$

where $L$ is the CONTROL VARIABLE. Once $V$ has been found, we use it to derive the optimal cumulative-dividends process $L^{*}=\left\{L^{*}(t), t \geq 0\right\}$.

What kind of properties should one expect $V$ to have? First, if $x$ equals zero the firm is liquidated and the shareholders receive the liquidation value, which is zero. In other words $V(0)=0$. The risk of bankruptcy implies that the marginal value of cash in the firm is high when $x$ is small. The said risk diminishes when $x$ grows, and since the only purpose cash serves in the firm is warding off bankruptcy, it is natural to expect that $x \mapsto V^{\prime}(x)$ is a decreasing mapping. We prove this in the following

\footnotetext{
4 The assumption of a zero liquidation value is made to simplify the exposition, and can be easily relaxed.

5 We are assuming here that there are no conflicts of interest between management and owners.
} 
Proposition 2.2 The mapping $x \mapsto V(x)$ is positive, increasing and concave.

Let us first examine the first-best case, where there are no financial frictions. In the said case the firm is never liquidated and its value at time 0 is simply the sum of the initial cash reserves and the expected present value of the future cashflows:

$$
V_{\mathrm{fb}}(x):=x+\frac{\mu}{r} \text {. }
$$

The implementation of the corresponding strategy does not have a unique solution (see, e.g. Sect. 3.3 for an implementation using equity issuance). This is a consequence of the celebrated Modigliani-Miller theorem, which for our purposes could be stated as

In the absence of financial frictions, the firm's market value is independent of its dividend policy.

We now go back to the case with financial frictions. The risk neutrality of the firm's owners yields a non-standard stochastic control problem: The value function satisfies a Hamilton-Jacobi-Bellman (HJB) variational inequality, where an ordinary differential equation describes its behavior whenever no distribution of dividends takes place, and a complementarity condition describes the set where it occurs. This embeds the manager's problem into the SINGULAR CONTROL framework.

Theorem 2.3 If the mapping $x \mapsto V(x)$ is twice continuously differentiable, then the following HJB variational inequality holds for all $x \geq 0$ :

$$
\max \left\{\frac{\sigma^{2}}{2} V^{\prime \prime}+\mu V^{\prime}-r V, 1-V^{\prime}\right\}=0,
$$

together with the boundary condition $V(0)=0$.

The left-hand term inside the maximization in Expression (3) is a standard HJB equation, and it represents the first order conditions arising from the Itô formula. Intuitively, the complementary condition $1-V^{\prime} \leq 0$ works as follows: We have that $V^{\prime}\left(0_{+}\right)>1$, otherwise for small levels of $x$, cash reserves retained in the firm would be worth, from the risk-neutral shareholders' perspective, the same or less than they would if distributed as dividends, which would bring us back to the first-best case. By concavity, the mapping $x \mapsto V^{\prime}(x)$ is decreasing and whenever $V^{\prime}(x)$ equals 1, additional funds are worth to shareholders equally in the firm or paid out. There will be a unique cash-reserves level $x^{*}$ such that no distribution of dividends takes place as long as $R^{L}<x^{*}$, and whenever $R^{L} \geq x^{*}$ dividends are distributed. This level is precisely the smallest $x$ such that the condition $V^{\prime}(x)=1$ is satisfied. In the sequel we refer to $x^{*}$ as the DIVIDEND BOUNDARY.

It should be noted that Theorem 2.3 only provides necessary conditions for optimality. Below we construct a candidate for the value function that satisfies the conditions stated in the theorem. We then verify that our candidate is indeed the sought-after value function. To this end, let us assume we tried to guess the dividend boundary $x^{*}$. For $x$ in $\left(0, x^{*}\right)$ the value function must satisfy both $V^{\prime}(x)>1$ and the linear, second order ordinary differential equation $\frac{\sigma^{2}}{2} V^{\prime \prime}+\mu V^{\prime}-r V=0$, whose general solution is

$$
U(x)=b_{1} e^{r_{1} x}+b_{2} e^{r_{2} x}, \quad \text { where } r_{i}:=\frac{-\mu+(-1)^{i} \sqrt{\mu^{2}+2 \sigma^{2} r}}{\sigma^{2}} .
$$

Note that $r_{1}<0<r_{2}$ and $\left|r_{1}\right|>\left|r_{2}\right|$. For each $x^{*}>0$ we define $V_{x^{*}}$ as the particular solution to Eq. (4) that satisfies $V_{x^{*}}^{\prime}\left(x^{*}\right)=1$ and $V_{x^{*}}(0)=0$. It is not complicated to show that

$$
V_{x^{*}}(x)=\frac{1}{r_{2} e^{r_{2} x^{*}}-r_{1} e^{r_{1} x^{*}}}\left(e^{r_{2} x}-e^{r_{1} x}\right) .
$$


Since the factor $e^{r_{2} x}-e^{r_{1} x}$ is non-negative, finding a candidate $\tilde{V}$ for the value function among the family $\left\{V_{x^{*}}\right\}_{x^{*} \geq 0}$ reduces to minimizing the mapping

$$
x^{*} \mapsto r_{2} e^{r_{2} x^{*}}-r_{1} e^{r_{1} x^{*}},
$$

which is positive and convex. Using the first-order conditions for optimality we find

$$
x^{*}=\frac{2}{r_{1}-r_{2}} \log \left(-\frac{r_{2}}{r_{1}}\right)>0 .
$$

Furthermore, this choice of $x^{*}$ implies that $V_{x^{*}}^{\prime \prime}\left(x^{*}\right)=0$ and $V_{x^{*}}\left(x^{*}\right)=\frac{\mu}{r}$. To verify that $V^{\prime}>1$ on $\left(0, x^{*}\right)$ we observe that such condition is equivalent to the mapping $x \mapsto$ $r_{2} e^{r_{2} x}-r_{1} e^{r_{1} x}$ being decreasing. This, however, holds whenever

$$
\left(\frac{r_{2}}{r_{1}}\right)^{2} e^{\left(r_{2}-r_{1}\right) x}<1 \Leftrightarrow x<x^{*} .
$$

On $\left[x^{*}, \infty\right)$ the value function is linear and has derivative equal to 1 . Since $\tilde{V}$ must be continuous we have

$$
\tilde{V}(x)=\left(x-x^{*}\right)+\frac{\mu}{r} .
$$

Notice this means that for $x>x^{*}$, the project's value is the sum of an immediate dividend of size $x-x^{*}$ plus the discounted value of a non-defaultable bond paying a continuous dividend $\mu d t$ per unit of time. The fact that the second derivative of $\tilde{V}$ at $x^{*}$ exists (and in this case equals zero) is commonly referred to as a SUPER- CONTACT CONDITION. It appears repeatedly in the stochastic control literature, where it is deemed a necessary condition for optimality (see, for example, the works of Dumas [7] and Højgaard and Taksar [9]). Our candidate for the value function is

$$
\tilde{V}(x)= \begin{cases}V_{x^{*}}(x), & x \in\left[0, x^{*}\right) ; \\ \left(x-x^{*}\right)+\frac{\mu}{r}, & x \in\left[x^{*}, \infty\right) .\end{cases}
$$

An observation that will prove handy in Sect. 3 is that since $V^{\prime}(x)>1$ for $x<x^{*}$ and $V\left(x^{*}\right)=\frac{\mu}{r}$ then

$$
x^{*}<\frac{\mu}{r} \text {. }
$$

\subsection{The optimal dividend-distribution strategy}

With the target cash level $x^{*}$ at hand, we may now describe the structure of the optimal dividend-distribution strategy $\left\{L^{*}(t)\right\}_{t \geq 0}$. Intuitively speaking, whenever the level of cash reserves $x$ grows over $x^{*}$, the difference $x-x^{*}$ must be paid out to the shareholders. On the interval $\left(0, x^{*}\right)$, however, the firm's reserves evolve according to an Itô process that has almost-surely continuous paths. This implies that, for any time $t>0$, the optimal dividend-distribution strategy must reflect the cash-reserves process $R^{L^{*}}$ downwards at the level $x=x^{*}$. The said reflection must be of an infinitesimal magnitude, given that for $x<x^{*}$ we have $V^{\prime}(x)>1$, and then shareholders prefer to keep the cash in the firm. The problem of governing an Itô diffusion so as to keep it constrained to the interval $[0, \infty)$ by means of an instantaneous reflection was first studied by Skorokhod in [24]. ${ }^{6}$ In our case we want to constrain $R^{L^{*}}$ to $\left(-\infty, x^{*}\right]$, but we kill the process at time $\tau_{L^{*}}$. To formalize this, let us

6 Up-to-date treatises on reflection problems can be found in the works of Bass and Hsu [4] and Kruk et al. [16]. 
consider the so-called Skorokhod problem on $\left(-\infty, x^{*}\right]$ that consists of finding two adapted processes $\left\{\left(R^{*}(t)\right\}_{t \geq 0} \text { and }\left\{L^{*}(t)\right)\right\}_{t \geq 0}$ that solve

$$
\begin{aligned}
& R^{*}(t)=x+\int_{0}^{t} \mu d s+\int_{0}^{t} \sigma d W(s)-L^{*}(t), 0<t \leq \tau_{L}, \\
& R^{*}(t) \leq x^{*}, 0<t \leq \tau_{L^{*}}, \\
& \int_{0}^{\tau_{L}} \mathbb{1}_{\left\{R^{*}(t)<x^{*}\right\}} d L^{*}(t)=0,
\end{aligned}
$$

where $\mathbb{1}_{\{\cdot\}}$ is the zero-one indicator function. Eq. (7) describes the firm's net earnings as had been specified. Expression (8) yields the constraining effect of the reflective barrier. Notice that it is implicitly assumed that $x \leq x^{*}$. The solution to the Problem (7)-(9) can be found, for instance, in Karatzas and Shreve [14] (Chapter 3, Lemma 6.14). The process $L^{*}=\left\{L^{*}(t)\right\}_{t \geq 0}$ is the local time of $R^{*}=\left\{R^{*}(t)\right\}_{t \geq 0}$ at level $x^{*}$. The impact of $L^{*}$ on the dynamics of $R^{*}$ is to reflect the latter so that it remains under $x^{*}$. If it were not the case that $x \leq x^{*}$, we would have to redefine $L^{*}$ at $t=0$ to be $L(0)=x-x^{*}$. For later dates $L^{*}$ would remain unchanged. From Eq. (9) we see that the mass of the measure $d L^{*}$ is carried by the sets $\left\{R^{*}=x^{*}\right\}$; thus $L^{*}$ is inactive whenever $R^{*}<x^{*}$. This coincides with our saying that no dividend distribution takes place before the target level $x^{*}$ is reached.

To finalize our characterization of the solution to the manager's problem we must verify that $\tilde{V}$ is indeed the value function, which is done in the following theorem.

Theorem 2.4 Let $\mu, \sigma$ and $r$ be greater than zero and let $x^{*}$ be the corresponding target level. If $\tilde{V}$ is the candidate value function defined in Eq. (5) and $\left\{L^{*}(t)\right\}_{t \geq 0}$ and is the local time of $\left\{R^{*}(t)\right\}_{t \geq 0}$ at level $x^{*}$, then for all $x \geq 0$

$$
\tilde{V}(x)=V^{L^{*}}(x)=V(x) .
$$

\subsection{Economic implications}

Let us further analyze the target level

$$
x^{*}=\frac{2}{r_{1}-r_{2}} \log \left(-\frac{r_{2}}{r_{1}}\right)>0
$$

and how it depends on the expectation and the variance of the firm's net earnings. Substituting the values for $r_{1}$ and $r_{2}$, it is not complicated to show that $x^{*}$ is increasing in $\sigma$. This is not surprising, and it may be interpreted as the manager being more conservative when facing a riskier project. What may come across as unexpected is that $x^{*}$ is single peaked in $\mu$. The target ratio decreases to zero as $\mu$ increases, since a highly profitable project requires less precautionary reserves. When $\mu$ is small, however, the target ratio must be adjusted downwards to increase the probability that the cash reserves will hit it, even if this implies a higher risk of default.

When it comes to financial frictions, their cost may be proxied by the difference between the first-best solution $V_{\mathrm{fb}}$ and $V$. Notice that $V_{\mathrm{fb}}\left(x^{*}\right)-V\left(x^{*}\right)=x^{*}$, which allows us to readily assess the gains from risk management. Namely, suppose one could reduce $\sigma$ to $\sigma-\Delta \sigma$. In the absence of frictions this has no impact on the value of the firm, since $V_{\mathrm{f}}$ is independent 
of $\sigma$. In our context, however, reducing $\sigma$ reduces the cost of financial frictions, ${ }^{7}$ since

$$
x^{*}(\sigma)>x^{*}(\sigma-\Delta \sigma) .
$$

\section{Equity issuance}

In the model introduced in Sect. 2, the sole instrument the manager had at his disposal to control the firm's level of cash reserves was the distribution of dividends. In this section, which is based on Décamps et al. [6], we enlarge the set of controls and introduce the possibility of equity issuance. It is well known that this is a costly process. For example, Franks and Sanzhar report in [8], when studying an UK sample of distressed equity issuers from 1989 to 1998, that the costs of underwriting new equity are $12.74 \%$ of the market value of existing equity. In practice, publicly traded companies typically issue new shares by organizing seasoned equity offerings (SEOs), which are quite costly operations. Costs include the fees paid to the investment banks managing the issuance, as well as other direct expenses, such as legal and auditing costs. Ross, Westerfield, and Jordan estimate in [23] the total direct costs of SEOs by U.S. corporations from 1990 to 2003 to be, on average, $6.72 \%$ of gross proceeds, rising to $12.88 \%$ for issuances of less than $\$ 10$ million. As a consequence, new issuances of equity are relatively infrequent and typically involve substantial amounts. For this reasons, we focus on the case of a fixed cost of equity issuance. Regarding mathematical methodologies, the proofs of several results in Sect. 2 can be easily adapted for use in the current section. Whenever such is the case, we avoid providing a full new proof and only mention in the text what the corresponding modifications should be.

\subsection{The structure of the equity-issuance process}

We keep the structure of the net earnings introduced in Sect. 2 unchanged, but now assume that the manager has the choice of issuing new equity. Due to the fact that the firm's size is fixed, equity issuance is done, should it occur at all, with the sole purpose of bolstering the firm's reserves. There is a fixed cost $\kappa \geq 0$ of issuance. In other words, if the manager decides to inject $x$ units of cash into the firm, $x+\kappa$ must be raised. An optimal policy must determine when is it that issuance takes place (if at all), as well as the issuance level. Observe that the cost structure readily implies that on any finite interval $\left[t_{0}, t_{1}\right]$ only a finite number of issuances may take place; otherwise, costs would become infinite in finite time, which would not be feasible. In order to describe in full generality the possible times and levels of equity issuance we introduce the following definition.

Definition 3.1 Let $\left\{\lambda_{i}\right\}_{i=1}^{\infty}$ be a sequence of strictly increasing $\mathcal{F}$-stopping times. Let $\left\{x_{i}\right\}_{i=1}^{\infty}$ be a sequence of non-negative random variables such that $x_{i} \in \mathcal{F}_{\lambda_{i}}$. Define

$$
J(t):=\sum_{i=1}^{\infty} x_{i} \mathbb{1}_{\left\{t \geq \lambda_{i}\right\}} \quad \text { and } \quad J_{\kappa}(t):=\sum_{i=1}^{\infty}\left(x_{i}+\kappa\right) \mathbb{1}_{\left\{t \geq \lambda_{i}\right\}},
$$

$J$ will be referred to in the sequel as a CUMULATIVE ISSUANCE STRATEGY. Further, let $L$ be a cumulative dividend process. For a given initial level of cash reserves $x$, we say that the pair $\pi:=(L, J)$ is admissible if the following conditions hold:

7 The implications of this observation in terms of risk-management are explored by Rochet and Villeneuve in [22]. 
(1) The process $R^{\pi}$, whose dynamics are given by

$$
d R^{\pi}(t)=\mu d t+\sigma d W(t)-d L(t)+d J(t), \quad R(0)=x
$$

is a.s. non-negative.

(2) Shareholder value is non-negative, ${ }^{8}$ i.e.

$$
V_{\kappa}^{\pi}(x):=\mathbb{E}\left[\int_{0}^{\infty} e^{-r t}\left(d L(t)-d J_{\kappa}(t)\right)\right] \geq 0 .
$$

Observe that any non-trivial cumulative issuance strategy $J$ induces a jump in $R^{\pi}$ of magnitude $x_{i}$ at date $t=\lambda_{i}$. Stochastic control problems where the control may generate jumps in the state variable are referred to as IMPULSE CONTROL PROBLEMS.

We denote by $\mathscr{A}_{f}$ the set of all admissible pairs $(L, J)$. An important observation is that for large values of $\kappa$, relative to the firm's expected profitability $\mu / r$, it may very well happen that $\mathscr{A}_{f}=\emptyset$. In such situation the firm defaults whenever its cash reserves hit zero, and we revert to the model in Sect. 2. The critical value of $\kappa$ above which there are no new equity issuances is characterized in Sect. 3.3. For the time being we look at the case where $\kappa$ is small and new equity issuances take place. The following result characterizes the optimal issuance times. It formalizes the intuition that, given that fresh equity is not used to expand the firm but simply to ward bankruptcy off, the manager should delay issuance for as long as possible.

Proposition 3.2 Assume that for some $\mu, r, \sigma>0$ the issuance cost $\kappa$ is such that the acceptance set $\mathscr{A}_{f}$ is non-empty. Let $\pi=(L, J) \in \mathscr{A}_{f}$ and assume that for some $\lambda_{i}$ it holds that $R^{\pi}\left(\lambda_{i}\right)>0$, then there exist $\tilde{\pi} \in \mathscr{A}_{f}$ such that $V_{\kappa}^{\pi}(x) \leq V_{\kappa}^{\tilde{\pi}}(x)$ for all $x>0$.

We have that for any optimal dividend-issuance strategy, equity issuance occurs, if ever, exclusively when the firm's reserves hit zero. This allows us to define the family of stopping times when fresh equity could be issued: For a given cumulative dividend strategy $L$ and initial cash reserves level $x>0$, let us define the sequence $\left\{\tau_{i}^{L}\right\}_{i=1}^{\infty}$ of $\mathcal{F}$-stopping times as follows:

$$
\begin{aligned}
& \tau_{1}^{L}:=\inf \left\{t>0 \mid R_{1}^{L}(t) \leq 0\right\}, \quad \text { where } R_{1}^{L} \text { solves } d R_{1}^{L}(t)=\mu d t+\sigma d W(t)-d L(t), \\
& R_{1}^{L}(0)=x ;
\end{aligned}
$$

for $i>1$ we define $\tau_{i}^{L}:=\inf \left\{t>\tau_{i-1} \mid R_{i}^{L}(t) \leq 0\right\}$, where $R_{i}^{L}$ solves

$$
d R_{i}^{L}(t)=\mu d t+\sigma d W(t)-d L(t), \quad R_{i}^{L}\left(\tau_{i-1}\right)=\hat{x} .
$$

The fact that the model is stationary, implies that, whenever an equity issuance takes place, it involves the same amount $\hat{x}>0$. Let us define the auxiliary process $I^{L}:=\left\{I^{L}(t)\right\}_{t \geq 0}$ via

$$
I^{L}(t)= \begin{cases}0, & t \in\left[0, \tau_{1}\right) \\ i-1, & x \in\left[\tau_{i-1}, \tau_{i}\right) .\end{cases}
$$

The cumulative issuance process corresponding to an issuance level $\hat{x}$ and $\operatorname{cost} \kappa$ is then

$$
G^{L}(t)=(\hat{x}+\kappa) I^{L}(t) .
$$

\footnotetext{
8 In order to facilitate comparative statics at the level of value functions for different levels of $\kappa$, we have chosen to indicate the latter as a subindex.
} 
Notice that as a consequence of Proposition 3.2, the set of admissible strategies may be redefined as

$\mathscr{A}_{f}^{0}:=\left\{\pi=(L, \hat{x}) \mid L(t) \in \mathcal{F}_{t}, L\right.$ is non-decreasing and càdlàg, $\hat{x} \geq 0$ and $\left.V^{\pi}(x) \geq 0\right\}$.

If the manager chooses the strategy $\pi=(L, \hat{x})$, then the dynamics of the cash-reserves process are

$$
d R^{\pi}(t)=\mu d t+\sigma d W(t)-d L(t)+\hat{x} d J(t), \quad R^{\pi}(0)=x .
$$

The corresponding shareholder value is

$$
V_{\kappa}^{\pi}(x)=\mathbb{E}\left[\int_{0}^{\infty} e^{-r t}(d L(t)-d G(t))\right]=\mathbb{E}\left[\int_{0}^{\infty} e^{-r t}(d L(t)-(\hat{x}+\kappa) d J(t))\right] .
$$

As before, the manager's objective is to find the strategy that implements the optimal value function:

$$
V_{\kappa}(x):=\sup _{\pi \in \mathscr{A}_{f}^{0}} V_{\kappa}^{\pi}(x), \quad x \in \mathbb{R}_{+} .
$$

\subsection{The impact of equity-issuance on the value function}

Given that the difference between the model with infinite frictions and the current one is the manager's option to issue fresh equity, and that this corresponds to a condition on the value function at $x=0$, it should come as no surprise that $V_{\kappa}$ is still twice continuously differentiable and satisfies the HJB Variational Inequality (3). The target cash level at which dividends are distributed and where the inequality $V_{\kappa}^{\prime} \geq 1$ becomes binding will be denoted by $x_{\kappa}^{*}$. The distribution boundary depends, in general, on the fixed-cost level $\kappa$. The boundary condition at $x=0$ must be determined endogenously. Namely, we must determine what the optimal level of new issuance should be, which is done by finding

$$
\max _{x \geq 0}\left\{V_{\kappa}(0+x)-x-\kappa\right\} \text {. }
$$

The expression $V_{\kappa}(0+x)-x-\kappa$ is the value of the firm if it is refinanced by an amount $x$ when its cash reserves hit zero, net of the refinancing costs. Using the first-order conditions (keep in mind that $V_{\kappa}$ is concave) we find that any maximizing $\hat{x}$ must satisfy $V_{\kappa}^{\prime}(\hat{x})=1$. In other words $\hat{x}=x_{\kappa}^{*}$, which yields the following result.

Proposition 3.3 Let $\mu, r, \sigma$ and $\kappa$ be greater than zero. There exists $x_{\kappa}^{*} \geq 0$ such that, if the value function $V_{\kappa}$ is twice continuously differentiable, then the following relations hold:

(i) $\frac{\sigma^{2}}{2} V_{\kappa}^{\prime \prime}+\mu V_{\kappa}^{\prime}-r V_{\kappa}=0$ and $V_{\kappa^{\prime}}<1$ on $\left(0, x_{\kappa}^{*}\right)$;

(ii) $V_{\kappa}^{\prime} \equiv 1$ on $\left(x_{\kappa}^{*}, \infty\right)$;

(iii) $V_{\kappa}(0)=\max \left\{V_{\kappa}\left(x_{\kappa}^{*}\right)-x_{\kappa}^{*}-\kappa, 0\right\}$.

The boundary condition at zero captures the fact that the optimal level of equity issuance is precisely the cash target, ${ }^{9}$ unless $\kappa$ is prohibitively high, in which case the firm defaults whenever its cash reserves hit zero.

Combining the super-contact condition $V_{\kappa}^{\prime \prime}\left(x_{\kappa}^{*}\right)=0$ with the smooth-pasting condition $V_{\kappa}^{\prime}\left(x_{\kappa}^{*}\right)=1$ yields the following candidate value function on $\left(0, x_{\kappa}^{*}\right)$ :

$$
\tilde{V}_{\kappa}(x)=\frac{1}{r_{1} r_{2}}\left(\frac{r_{2}^{2}}{r_{2}-r_{1}} e^{r_{1}\left(x-x_{\kappa}^{*}\right)}-\frac{r_{1}^{2}}{r_{2}-r_{1}} e^{r_{2}\left(x-x_{\kappa}^{*}\right)}\right),
$$

\footnotetext{
9 This is no longer true if issuing new equity also carries a proportional-cost component as in [6].
} 


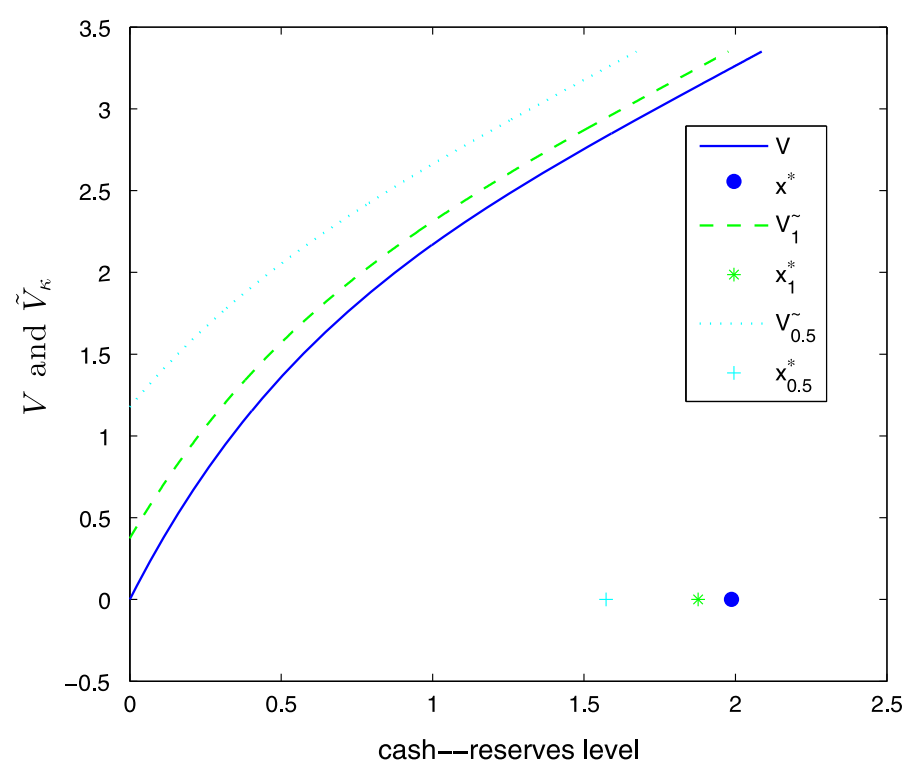

Fig. 1 The value function and distribution boundary for different levels of $\kappa$

where $r_{1}$ and $r_{2}$ are as in Eq. (4). It is still the case that $\tilde{V}_{\kappa}\left(x_{\kappa}^{*}\right)=\frac{\mu}{r}$, therefore the target cash level is found by solving

$$
\phi(x):=\frac{r_{2}^{2} e^{-r_{1} x}-r_{1}^{2} e^{-r_{2} x}}{r_{1} r_{2}\left(r_{2}-r_{1}\right)}=\frac{\mu}{r}-x-\kappa .
$$

We observe that the mapping $x \mapsto \phi(x)$ is decreasing, since $r_{1}<0<r_{2}$ and $r_{1} r_{2}\left(r_{2}-r_{1}\right)<$ 0 . For the target cash level $x^{*}$ of the infinite-frictions model, it holds that $\phi\left(x^{*}\right)=0$. This allows us to compare the target cash levels with and without the possibility of equity issuance, which we do in the following lemma.

Lemma 3.4 Let $\mu, r, \sigma$ and $\kappa$ be greater than zero and such that $\tilde{V}_{\kappa}(0)=\frac{\mu}{r}-x_{\kappa}^{*}-\kappa>0$. Further, let $x^{*}$ be the target cash level of the infinite-frictions model with parameters $\mu, r$ and $\sigma$, then $x_{\kappa}^{*}<x^{*}$.

In other words, whenever $\kappa$ is such that fresh equity is issued as the firm runs out of cash, then dividend distribution occurs sooner than in the basic model. The rationale for this is that dividend distribution is only postponed in order to ward off bankruptcy. Having a second vehicle to do this, namely the possibility to issue new equity, reduces the marginal value of cash in the firm, which in turn implies that the manager may lower the level of reserves at which dividends are paid out. In Fig. 1 we plot $\tilde{V}_{0.5}$ and $\tilde{V}_{1}$, together with the corresponding dividend-distribution barriers, for $\mu=0.7, \sigma=0.5$ and $r=0.2$. The value function and distribution boundary of the infinite-frictions case, with the same parameters, is also plotted.

\subsection{Between strategic default and the first-best}

Whenever the level of cash reserves reaches zero, the firm's manager will avoid issuing fresh equity and instead will allow the firm to default if $\frac{\mu}{r}-x_{\kappa}^{*}-\kappa \leq 0$. Observe that $\phi(0)$ coincides with $\tilde{V}_{\kappa}\left(x_{\kappa}^{*}\right)=\frac{\mu}{r}$ and $\lim _{x \rightarrow \infty} \phi(x)=\lim _{x \rightarrow \infty} \phi^{\prime}(x)=-\infty$. Put differently, 
Eq. (13) has a unique solution for any positive value of $\kappa$. Since the mapping $x \mapsto \frac{\mu}{r}-x-\kappa$ hits zero at $x=\frac{\mu}{r}-\kappa$ and $\phi\left(x^{*}\right)=0$, the system

$$
\left\{\begin{array}{l}
\phi(x)=\frac{\mu}{r}-x-\kappa \\
\frac{\mu}{r}-x-\kappa>0
\end{array}\right.
$$

has a solution if and only if $\frac{\mu}{r}-\kappa>x^{*}$ and we know from inequality (6) that $\frac{\mu}{r}>x^{*}$. These observations, together with arguments analogous to those found in the proof of Theorem 2.4, yield the following result.

Theorem 3.5 Let $\mu, r$ and $\sigma$ be greater than zero. There exists $\bar{\kappa} \geq 0$ such that if $\kappa \in[0, \bar{\kappa})$ then equity is issued at date $t$ if and only if $R^{\pi}(t)=0$. In this case the optimal strategy is $\pi^{*}=\left(x_{\kappa}^{*}, L^{*}\right)$, where $x_{\kappa}^{*}$ solves System (14) and $\left\{L^{*}(t)\right\}_{t \geq 0}$ is the local time of $\left\{R^{\pi^{*}}(t)\right\}_{t \geq 0}$ at level $x_{\kappa}^{*}$. In other words, for all $x \geq 0$ it holds that

$$
\tilde{V}_{\kappa}(x)=V^{\pi^{*}}(x)=V_{\kappa}(x) .
$$

On the other hand, if $\kappa>\bar{\kappa}$ then $\frac{\mu}{r}-\kappa<x^{*}$, equity is never issued, and we revert to the basic model where $V(0)=0$ and $x_{\kappa}^{*}=x^{*}$.

In the absence of frictions, i.e. when $\kappa=0$, the first-best is attainable: In this case $x^{*}=0$ solves System (14); thus, the firm does not keep any cash reserves and the dividend policy $d L(t)$ becomes irrelevant. Namely, cashflows, whether positive or negative, are absorbed by shareholders (dividends or new equity issuance).

\subsection{Stock-price dynamics}

Let the process $N=\{N(t)\}_{t \geq 0}$ represent the number of shares outstanding at date $t \geq 0$. As long as $R^{\pi}(0)>0$, we may assume without loss of generality that $N(0)=1$. Observe that $N(t)$ remains constant between any two consecutive issuance times $\tau_{i}<\tau_{i+1}$. In the sequel, the process $S=\{S(t)\}_{t \geq 0}$ represents the price per share of the firm's stock, so the firm's market capitalization is given at each date $t \geq 0$ by $N(t) S(t)$. As a consequence of the fact that issuance and dividend decisions at any time $t>0$ depend exclusively on the level of cash reserves accumulated by the firm, the market capitalization of the firm satisfies

$$
N(t) S(t)=V_{\kappa}\left(R^{*}(t)\right), \quad t>0 .
$$

Since dividends are only distributed when $R^{*}(t) \geq x_{\kappa}^{*}$ and new equity is only issued when $R^{*}(t)=0$, as long as $0<R^{*}(t)<x_{\kappa}^{*}$ we may derive the dynamics of the stock price from Eq. (15) and the Itô formula. Namely,

$$
d(N(t) S(t))=N(t) d S(t)=d V_{\kappa}\left(R^{*}(t)\right)=V_{\kappa}^{\prime}\left(R^{*}(t)\right) d R^{*}(t)+\frac{\sigma^{2}}{2} V_{\kappa}^{\prime \prime}\left(R^{*}(t)\right) d t,
$$

which using again Eq. (15) may be rewritten as

$$
\frac{d S(t)}{S(t)}=\frac{\left[\mu V_{\kappa}^{\prime}\left(R^{*}(t)\right)+\left(\sigma^{2} / 2\right) V_{\kappa}^{\prime \prime}\left(R^{*}(t)\right)\right] d t+\sigma V_{\kappa}^{\prime}\left(R^{*}(t)\right) d W_{t}}{V_{\kappa}\left(R^{*}(t)\right)} .
$$

On $\left(0, x_{\kappa}^{*}\right)$ it holds that $\frac{\sigma^{2}}{2} V_{\kappa}^{\prime \prime}+\mu V_{\kappa}^{\prime}-r V_{\kappa}=0$, hence

$$
\frac{d S(t)}{S(t)}=r d t+\sigma(t, S(t)) d W(t)
$$


where

$$
\sigma(t, S(t)):=\sigma \frac{V_{\kappa}^{\prime}\left(R^{*}(t)\right)}{N(t) S(t)}=\sigma \frac{V_{\kappa}^{\prime}\left(R^{*}(t)\right)}{V_{\kappa}\left(R^{*}(t)\right)} .
$$

Given that $V_{\kappa}$ is an increasing function, Eq. (15) implies that, between issuance occurrences, $S(t)$ and $R^{*}(t)$ are comonotonic. Hence, $s \mapsto \sigma(t, s)$ is a decreasing mapping for all $t \geq 0$. In other words, the stock of a highly-capitalized firm is less volatile than that of a lesscapitalized one. It is important to point out that, since dividend distribution keeps $R^{*} \leq x_{\kappa}^{*}$, the stochastic volatility $\sigma(t, S(t))$ is bounded below by $\frac{\sigma r}{\mu}$. This value coincides with the (constant) volatility on the firm's stock when financial frictions disappear. Equation (15) also allows us to compute the ratio $k(\lambda)$ of new shares to outstanding shares whenever equity issuance takes place. Indeed, let us consider the $n$-th issuance time $\lambda_{n}$, then

$$
k\left(\lambda_{n}\right)=\frac{N\left(\lambda_{n}\right)-N\left(\lambda_{n}-\right)}{N\left(\lambda_{n}\right)}=\frac{V_{\kappa}^{\prime}\left(x_{\kappa}^{*}\right)-V_{\kappa}^{\prime}(0)}{V_{\kappa}^{\prime}\left(x_{\kappa}^{*}\right)} .
$$

This shows that a constant dilution factor affects individual share prices each time issuance occurs. Finally, we observe that, since $N(t)$ is an increasing process (there are no share buybacks) and dividend distribution keeps $R^{*} \leq x_{\kappa}^{*}$, the stock price in this model is bounded from above.

\section{A model of bank portfolio choice}

The model presented in this section shares many mathematical features with the ones presented previously. The focus, however, switches from liquidity management in a firm with fixed expected profitability to a portfolio-decision problem à la Merton (see, e.g. Merton's paper [17]) from the point of view of a bank. More specifically, we study the optimal decisions of the manager of a bank whose balance sheet at date $t$ is the following:

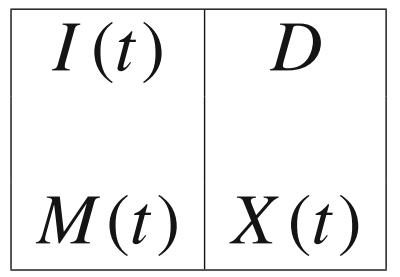

$D$ represents the volume of retail deposits that the bank has collected, which are remunerated at the risk free rate $r>0 . M(t)$ is the level of cash reserves in the bank, which also earn interest $r$. We shall see in Observation 4.4 below, that in this model the bank never defaults on its debt, which justifies the assumption that its debt bears no default spread. $X(t)$ is the book-value of equity and will play the role of the state variable (instead of the reserves level, as done in the previous sections). Finally, $I(t)$ represents the investment in a risky asset, which returns

$$
\mu d t+\sigma d W(t)
$$

per unit of investment. As soon as $X(t) \leq 0$, the bank is liquidated at no cost, and $D$ is repaid to the depositors. The shareholders walk away empty-handed in the event of bankruptcy. 
Financial frictions are captured by the fact that no additional debt or equity may be issued. Therefore, the bank's investment strategies must be self-financing.

\subsection{Setting up the stochastic control problem}

As in the previous sections, the bank's manager decides on the cumulative dividenddistribution process $L=\{L(t), t \geq 0\}$. Here, however, he must also choose the INVESTMENT STRATEGY $I=\{I(t), t \geq 0\}$. The bank's balance sheet may be continuously rebalanced at no cost, but at every date $I(t) \in[0, X(t)+D]$ must hold. This restriction implies that the set of all admissible policies, which will be again denoted by $\mathscr{A}$, depends on the current book value of the bank's equity. The latter evolves according to the following stochastic differential equation:

$$
d X(t)=\underbrace{I(t)(\mu d t+\sigma d W(t))}_{\text {cashflows from risky asset }}+\underbrace{r(M(t)-D) d t}_{\text {interest earned/paid }}-\underbrace{d L(t)}_{\text {dividends distr. }}, \quad X(0)=x .
$$

Using the BALANCE- SHEET EQUATION $X(t)+D=I(t)+M(t)$ we may rewrite $d X(t)$ as

$$
d X(t)=I(t)(\mu d t+\sigma d W(t))+r(X(t)-I(t)) d t-d L(t), \quad X(0)=x,
$$

and we define $\tau:=\inf \{t \geq 0 \mid X(t) \leq 0\}$. The manager's problem is to maximize shareholder value:

$$
V(x)=\max _{(I, L) \in \mathscr{A}} \mathbb{E}_{x}\left[\int_{0}^{\tau} e^{-\rho t} d L(t)\right],
$$

where $\rho>\mu$ is the shareholders' discount rate.

Observation 4.1 If the shareholders discounted the future at a rate smaller than $\mu$, then the manager's problem would lack a well defined solution. To see this choose $\delta \in(0, \mu-\rho)$ and set

$$
L(t)=\int_{0}^{t} \delta X(t) d t, \quad I(t)=X(t)
$$

This would result in

$$
d X(t)=X(t)((\mu-\delta) d t+\sigma d W(t)) \Rightarrow \tau=\infty
$$

and

$$
V(x) \geq \delta x \int_{0}^{\infty} e^{(\mu-\rho-\delta) t} d t=\infty
$$

\subsection{The value function and the first-best}

An additional assumption on the model's parameters is that $\mu$ must be strictly greater than $r$. This implies that, in the absence of retail deposit financing, shareholders would prefer to consume immediately instead of investing in the risky asset. Being leveraged, however, allows the shareholders to make a profit. In particular, if we were to disregard the restriction that $t \mapsto L(t)$ must be non-decreasing, then we could parallel Sect. 2.2 and obtain the first-best outcome as follows: Hold no cash reserves; set $I(t) \equiv D$; offset profits/losses via payments to/from the shareholders. The resulting value function would be 


$$
V_{\mathrm{FB}}(x)=x+\frac{\mu-r}{\rho} D .
$$

Notice that the first-best strategy still consists of immediate consumption of all existing reserves, but the second term now corresponds to the expected present value of investing $D$ in the risky technology. The term $\mu-r$ stems from the fact that interest accrues in favour of the depositors at a rate $r$.

We should again have a concave, twice continuously differentiable value function and a dividend-distribution barrier $x_{1}^{*}$ that coincides with the point where the marginal value of equity equals one. In the current set up, though, the structure of the value function on $\left[0, x_{1}^{*}\right]$ should also take into account the investment strategies for different levels of equity value. In order to study this, let us define the operator

$$
L(I) V(x):=-\rho V(x)+V^{\prime}(x)(r x+I(\mu-r))+\frac{\sigma^{2} I^{2}}{2} V^{\prime \prime}(x),
$$

then we have the following verification result:

Theorem 4.2 Assume there exists a concave, twice continuously differentiable function $U$ and a constant $x_{1}^{*}$ such that

$$
\begin{array}{ll}
\max _{0 \leq I \leq X+D} L(I) U(x)=0 & \text { and } U^{\prime}(x)>1 \text { for all } x<x_{1}^{*} \text { and } \\
\max _{0 \leq I \leq X+D} L(I) U(x)<0 & \text { and } U^{\prime}(x)=1 \text { for all } x \geq x_{1}^{*}
\end{array}
$$

together with the initial condition $U(0)=0$. Then $U=V$.

The proof of Theorem 4.2 is an adaptation of that of Theorem 2.4 (we refer the reader to [21], p. 235 for details). The presence of the Neumann condition $U^{\prime}\left(x_{1}^{*}\right)=1$ yields that the optimal dividend-distribution strategy $L^{*}$ coincides with the local time of the process $X$ at level $x_{1}^{*}$. In terms of the investment strategy, we have that in general there will be a point $0<x_{0}^{*}<x_{1}^{*}$ such that the maximization problem

$$
\max _{I \in[0, x+D]} L(I) V(x)
$$

has an interior solution (and $\left.V^{\prime}>1\right)$ for all $x \in\left(0, x_{0}\right)$. Notice that this implies that if the value of equity is small enough, then $I(t)$ is strictly smaller than $X(t)+D$ which in turn means that $M(t)$ is greater than zero. In other words, for low levels of capitalization, the bank holds a positive level of reserves as a precautionary measure. On $\left(0, x_{0}\right)$ we may find $I^{*}$ using the first order condition

$$
\frac{\partial}{\partial I} L(I) V(x)=0
$$

which yields

$$
I^{*}(x)=\frac{\mu-r}{\sigma^{2}}\left(-\frac{V^{\prime}(x)}{V^{\prime \prime}(x)}\right) .
$$

Lemma 4.3 The general solution to the differential equation

$$
L\left(I^{*}(x)\right) V(x)=-\rho V(x)+r x V^{\prime}(x)-\frac{1}{2}\left(\frac{\mu-r}{\sigma}\right)^{2} \frac{\left(V^{\prime}(x)\right)^{2}}{V^{\prime \prime}(x)}=0, \quad V(0)=0
$$

is

$$
V(x)=K x^{\alpha},
$$


where $K>0$ and $\alpha \in(0,1)$ is the smallest solution to

$$
r \alpha^{2}-\left(r+\rho+\frac{1}{2}\left(\frac{\mu-r}{\sigma}\right)^{2}\right) \alpha+\rho=0 .
$$

Inserting Function (18) in Expression (17) we obtain

$$
I^{*}(x)=\frac{x}{k}, \quad \text { where } k \text { is defined as } \quad k:=\left(\frac{\sigma^{2}}{\mu-r}\right)(1-\alpha) .
$$

Observation 4.4 Since $d L(t)=0$ whenever $X(t)<x_{1}^{*}$, if we substitute $I^{*}(X(t))$ into $d X(t)$ we obtain that on $\left(0, x_{0}\right)$ the value of equity evolves according to

$$
d X(t)=\frac{X(t)}{k}[(\mu+r(k-1)) d t+\sigma d W(t)] .
$$

The factor $\mu+r(k-1)$ is strictly positive; thus, in this "distress" region $X(t)$ evolves like a geometric Brownian motion. This results in $\mathbb{P}\{X(t) \leq 0\}=0$ for all $t \geq 0$, hence $\mathbb{P}\{\tau=\infty\}=1$. In terms of the value function, this is reflected by the fact that, since $\alpha<1$, $V^{\prime}(0)=\infty$.

Lemma 4.5 On $\left(0, x_{0}^{*}\right)$ the target capital ratio $x / I^{*}(x)=k$ is less than one.

From Lemma 4.5 we have that on $\left(0, x_{0}^{*}\right)$ the manager's optimal strategy is to invest a multiple of the bank's equity in the risky technology. Moreover, $x_{0}^{*}$ is computed by solving $\frac{x_{0}^{*}}{k}=x_{0}+D$, which yields

$$
x_{0}^{*}=\frac{k D}{1-k} .
$$

For all $x \in\left(x_{0}^{*}, x_{1}^{*}\right)$ it holds that $V^{\prime}(x)>1$ and the maximization problem

$$
\max _{I \in[0, x+D]} L(I) V(x)
$$

has the corner solution $I^{*}(x)=x+D$. This is similar to the scenario presented in Sect. 2 in the sense that the portfolio weight on the risky asset is constant and maximal. On $\left(x_{0}^{*}, x_{1}^{*}\right)$ we have to solve

$$
\rho V(x)=(\mu x+(\mu-r) D) V^{\prime}(x)+\frac{\sigma^{2}}{2}(x+D)^{2} V^{\prime \prime}(x), \quad V^{\prime}\left(x_{1}^{*}\right)=1, V^{\prime \prime}\left(x_{1}^{*}\right)=0,
$$

and the choice of $x_{1}^{*}$ must be such that the resulting solution pastes in $\mathcal{C}^{2}$-fashion at $x_{0}^{*}$. First we require the following auxiliary result:

Lemma 4.6 For all $u \in \mathbb{R}_{+}$there exists a unique $V_{u} \in \mathcal{C}^{2}$ such that

$$
\rho V_{u}(x)=(\mu x+(\mu-r) D) V_{u}^{\prime}(x)+\frac{\sigma^{2}}{2}(x+D)^{2} V_{u}^{\prime \prime}(x), \quad V_{u}^{\prime}(u)=1, V_{u}^{\prime \prime}(u)=0 .
$$

From the conditions $V_{u}^{\prime}(u)=1$ and $V_{u}^{\prime \prime}(u)=0$ one can obtain that $V_{u}(u)=\frac{D(\mu-r)}{\rho}+\frac{\mu}{\rho} u .^{10}$ This determines the structure of the value function on $\left[x_{1}^{*}, \infty\right)$, and we have the following

10 Notice that the value of the bank at the target cash level depends on the value of the latter. This is in contrast with the cases studied in Sects. 2 and 3, where the firm's value at the candidates for the dividend-distribution barrier was always fixed. 


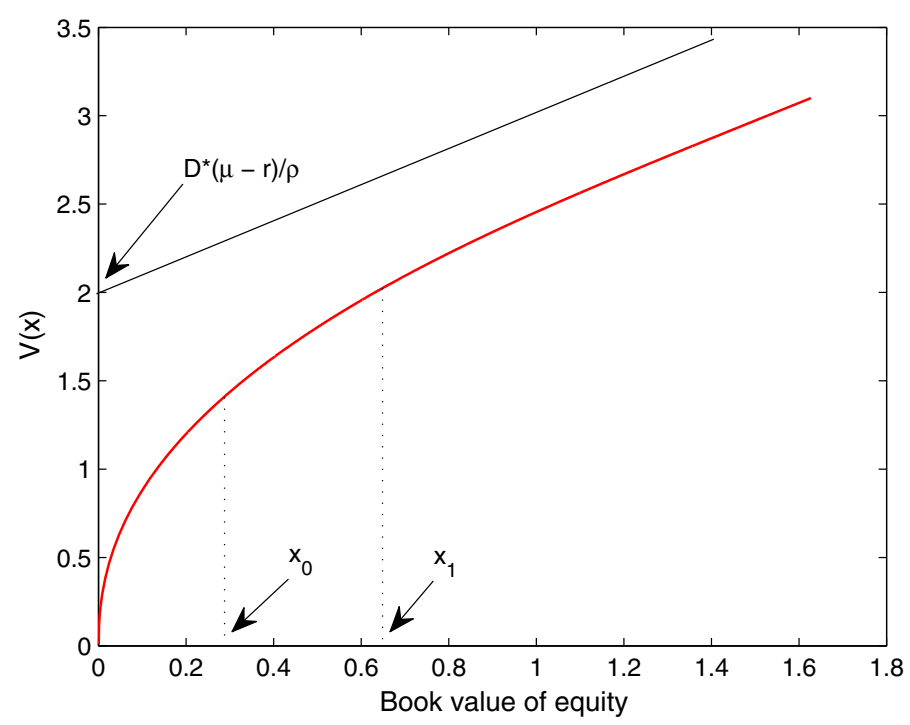

Fig. 2 The value function, the partial investment boundary and the dividend barrier

Theorem 4.7 There exists a unique $x_{1}^{*}$ such that the piecewise-defined function

$$
V(x):= \begin{cases}K x^{\alpha}, & x \in\left[0, x_{0}^{*}\right] ; \\ V_{x_{1}^{*}}(x), & x \in\left(x_{0}^{*}, x_{1}^{*}\right) ; \\ \left(x-x_{1}^{*}\right)+\frac{D(\mu-r)}{\rho}+\frac{\mu}{\rho} x_{1}^{*}, & x \in\left[x_{1}^{*}, \infty\right),\end{cases}
$$

is twice continuously differentiable (see Fig. 2).

We have established that the optimal investment policy of the bank was:

$$
I^{*}(x)=\min \left\{\frac{x}{k}, x+D\right\},
$$

i.e, invest a multiple $1 / k$ of the bank's equity $X(t)$, up to when this exhausts cash reserves. $k$ can be interpreted as a minimum capital ratio

$$
\frac{\text { equity }}{\text { investment }}
$$

as in bank solvency regulations, but here it is chosen by the shareholders.

Proposition 4.8 Ceteris paribus, the target capital ratio $k$ optimally chosen by the bank satisfies the following properties:

(1) It decreases with the cost of capital $(\rho-r)$.

(2) It increases with the volatility of assets $\sigma$.

(3) It is single peaked with respect to the expected excess return on assets $(\mu-r)$.

In the region where its investment policy is not constrained, the bank behaves like a Von Neumann-Morgenstern investor with Constant Relative Risk Aversion $1-\alpha$.

Proposition 4.9 Ceteris paribus, the risk aversion coefficient $1-\alpha$ of the bank satisfies the following properties: 
(1) It decreases with the cost of capital $(\rho-r)$.

(2) It decreases with the volatility of assets $\sigma$.

(3) It increases with the expected excess return on assets $(\mu-r)$.

\section{Further reading}

We conclude by looking at some ramifications of the methodologies presented above. These are mostly recent works that showcase how current this strand of the literature is. We encourage the interested reader to not only look at the papers we mention, but also at the references therein.

Insurance literature. Within the insurance literature, Højgaard and Taksar pioneered in [9] the use of an instantaneously-controlled Brownian motion to model the net earnings of an insurance firm. Here the manager controls the firm's risk exposure via proportional reinsurance, represented by the control variable $1-\alpha \in[0,1]$ that multiplies the firm's returns (if $\alpha=1$, the latter are as in Eq. (1)). The second control variable, the cumulative distribution of dividends, keeps the firm's cash reserves below or at the optimal dividend-distribution barrier $x^{*}$. The possibility of continuously fine-tuning the reinsurance level results in an additional (relative to the model in Sect. 2) boundary point $0<x^{* *}<x^{*}$. Below $x^{* *}$ there is partial reinsurance and on $\left(x^{* *}, x^{*}\right)$ there is none. In the partial-reinsurance region, the optimal choice $\alpha^{*}$ results in a reserves level that follows a geometric Brownian motion: The proportion of reinsurance tends to one as the reserves approach zero; thus preventing the firm from ever going bankrupt. ${ }^{11}$ In other words, in the world of Højgaard and Taksar a firm never defaults. This model was extended by Barth and Moreno-Bromberg in [2] to allow for costly equity issuance. The authors study the interaction between refinancing costs, liquidity management and risk-controlling strategies. In particular, they show that when issuance costs are proportional, using the recapitalization option is a dominant strategy. In contrast, in the fixed-cost scenario the highest cost level at which the firm issues fresh equity is characterized as a function of the firm's expected productivity and the volatility on its net returns. Rochet and Villeneuve analyze in [22] a similar model where only part of the Brownian risk can be hedged by the firm (this is done by introducing two independent Brownian motions, one driving the firm's net returns and a second one representing hedgeable risk). Furthermore, their model also incorporates a Poisson risk of fixed magnitude that can be insured against. The inclusion of this jump component results in an interesting departure from the purely Brownian models: The authors prove existence of a positive threshold $\underline{x}$ under which the firm chooses not to insure itself against the Poisson risk, since an immediate arrival would nonetheless prompt bankruptcy. This partially captures the idea that distressed firms act in a risk-loving way (they "gamble for resurrection" in the financial-literature jargon), which is absent in continuous-time models when the cash-reserves processes are continuous.

Liquidity management, investment and output decisions and capital structure. Milne and Roberston study in [18] extensions to the model we present in Sect. 2. Their aim is to understand how the threat of liquidation affects aspects of firm behavior, such as output and investment decisions. They find that only when the target cash level has been reached, the managerial choices of output and investment coincide with the first-best levels. This follows the fact that only at the said level of cash reserves is the firm locally unconstrained. Both output and investment are optimally adjusted downwards as the reserves' level decreases, which showcases that the firm, quoting the authors "becomes both locally more risk-averse

11 Notice the similarity with the model presented in Sect. 4. 
and more impatient as the level of cash-in-hand falls". As a side note, Milne's and Roberston's model with investment decisions has been recently used by Brunnermeier and Sannikov in [5] and by Isohätälä, Milne and Robertson in [11] in a macroeconomic setting with a financial sector. In terms of the interaction of liquidity management and capital structure of firms, Hugonnier and Morellec [10] and Klimenko and Moreno-Bromberg [15] study models where decisions on these two fronts are made simultaneously. In both papers the firm's reserves are exposed to a Poisson risk that models large, sudden losses. In [10] these losses are of random size and they represent, for example, defaults across the bank's loan portfolio. In this model bank shareholders have the possibility to recapitalize the bank when it runs out of cash. However, if the size of a large loss exceeds some critical threshold, liquidation is optimal. The authors jointly determine the maximum shortfall that shareholders are willing to cover and the target level of cash reserves. The focus in [15] is on the short-to-long-term debt structure of a financial firm, which, together with the coupon on long-term debt $r_{l}$, is determined endogenously. Short-term debt is fully collateralized and is, therefore, risk-less. There is a single downward jump in the firms' reserves that corresponds to the short-term debt holders stopping to roll their debt over (as a consequence of a sudden liquidity shock, for example). In the absence of refinancing possibilities, this may result in the firm going bankrupt and possibly being unable to honor its obligations towards long-term debt holders. The latter take this into account when accepting a certain coupon, hence there is a cost associated to issuing short-term debt that manifests itself through $r_{l}$. This impact might be mitigated through the proper use of a cash management policy, creating a link between the maturity structure of the firm and its liquidity-management policies.

Stochastic interest rates. Akyildirim et al. [1] and Jiang and Pistorius [13] have studied models where the short-rate is assumed to evolve according to a Markov chain $Z:=\{Z(t)\}_{t \geq 0}$. Jiang and Pistorius also allow for the firm's average profitability and the volatility on its earnings to depend on $Z$, in other words $\mu=\mu(Z(t))$ and $\sigma=\sigma(Z(t))$. This represents regime switching, and $Z$ is interpreted as the state of the economy. The model in [1] does not contemplate regime switching, but instead costly equity issuance is considered. Interestingly, when Akyildirim et al. allow for state-dependent issuance costs, they obtain a market-timing effect: When issuing costs are very high during recessions (so that shareholders refuse to recapitalize firms when they run out of cash) it becomes optimal to issue new equity in the good state even if the firm still has cash reserves, due to the fear that a recession might occur, leading to the forced closure of a profitable company. This is in contrast with the model in Sect. 3, where issuance occurred exclusively when the firm's reserves became non-positive. It should be noted that in the models considered in [1] and [13], a stochastic short-rate reflects on preferences as a discount rate, but no (stochastic) interest accrues on retained earnings. Taking an alternative approach, Barth, Moreno-Bromberg and Reichmann study in [3] a variation of the infinite-frictions model in Sect. 2 where interest accrues on retained earnings. The interest rate is stochastic (it is modeled by an Itô diffusion), but the discount rate of the investors is fixed. Their analysis is mostly numerical, employing finite-elements techniques to solve the partial differential equation with complementary conditions, analogous to Eq. (3), that arises in their setting.

\section{Appendix: Proofs}

Proof of Proposition 2.1 Let $L \in \mathscr{A}, 0<s<\tau_{L}$ and define for $l \geq s$ the auxiliary process

$$
H(l):=R^{L}(s)+\mu(l-s)+\sigma W(l-s) .
$$


Since $L$ is non-decreasing, we have that for all $l \geq s$ it holds that $M^{L}(l) \leq H(l)$. As a consequence, if we define

$$
\tau_{H}:=\inf \{l>s \mid H(l) \leq 0\}
$$

and choose $t>s$, then $\mathbb{P}\left\{\tau_{H}<t\right\} \leq \mathbb{P}\left\{\tau_{L}<t \mid R^{L}(s)\right\}$. A simple translation argument yields

$$
\mathbb{P}\left\{\tau_{H}<t\right\}=\mathbb{P}\left\{\tau_{\tilde{H}}<t-s\right\},
$$

where $\tilde{H}(l):=R^{L}(s)+\mu l+\sigma W(l)$ and $\tau_{\tilde{H}}$ is defined accordingly. Let $T=t-s$ and notice that

$$
\mathbb{P}\left\{\tau_{\tilde{H}}<t-s\right\}=\mathbb{P}\{\tilde{\tau}<T\},
$$

where $\tilde{\tau}$ is the first crossing time of the standard Browian motion $W$ and the linear boundary defined by $k(t)=\sigma^{-1}\left(R^{L}(s)+\mu t\right)$. It is well known (see, eg. Pötzelberger and Wang [19], p. 55) that

$$
\begin{aligned}
\mathbb{P}\{\tilde{\tau}<T\}= & 1-\Phi\left(\frac{\sigma^{-1}\left(R^{L}(s)+\mu T\right)}{\sqrt{T}}\right) \\
& +\exp \left\{-2 \mu R^{L}(s) / \sigma^{2}\right\} \Phi\left(\frac{\sigma^{-1}\left(\mu T-R^{L}(s)\right)}{\sqrt{T}}\right)>0
\end{aligned}
$$

which concludes the proof.

Proof of Proposition 2.2 For any $x>0$ the strategy $L$ defined via $L(0)=x$, and $d L(t) \equiv 0$ for $t>0$ results in $V^{L}(x)=x$; thus $V(x) \geq x$, which yields the required positivity and monotonicity properties of $V$. Next, consider the reserves levels $x_{1}, x_{2}>0$ and let $L_{1}$ and $L_{2}$ be two corresponding admissible strategies. Let $\lambda \in(0,1)$ and define

$$
\tilde{x}:=\lambda x_{1}+(1-\lambda) x_{2} \text { and } \tilde{L}:=\lambda L_{1}+(1-\lambda) L_{2} .
$$

Clearly $\tilde{L}$ is admissible and $d R^{\tilde{L}}=\lambda d R^{L_{1}}+(1-\lambda) d R^{L_{2}}$. Define $\tilde{\tau}:=\max \left\{\tau_{L_{1}}, \tau_{L_{2}}\right\}$, then, using the fact that the conditional-expectation operator is linear, we have

$$
V(\tilde{x}) \geq V^{\tilde{\pi}}(\tilde{x})=\lambda V^{\pi_{1}}\left(x_{1}\right)+(1-\lambda) V^{\pi_{2}}\left(x_{2}\right) .
$$

By definition, for all $\epsilon>0$ the strategy $L_{1}$ can be chosen such that $V^{L_{1}}\left(x_{1}\right) \geq V\left(x_{1}\right)-\epsilon / 2$, and analogously for $V\left(x_{2}\right)$. In other words, the expression

$$
V(\tilde{x}) \geq \lambda V\left(x_{1}\right)+(1-\lambda) V\left(x_{2}\right)-\epsilon
$$

holds for any positive $\epsilon$; thus the mapping $x \mapsto V(x)$ is concave.

Proof of Theorem 2.3 We first consider the condition on $V^{\prime}$. By definition, for any $h, y>0$ there exists a strategy $L_{y}$ such that $V^{L_{y}}(y) \geq V(y)-h^{2}$.

Let $0<h<x$ and construct a strategy $L$ by setting $L^{L}(t)=h+L^{L_{x-h}}(t)$, then

$$
V(x) \geq V^{L}(x)=h+V^{L_{x-h}}(x-h) \geq h+V(x-h)-h^{2},
$$

which is equivalent to

$$
\frac{V(x)-V(x-h)}{h} \geq 1-h .
$$


By the differentiability of $V$, we may let $h$ go to zero and conclude that $V^{\prime}(x) \geq 1$. Next, fix a strategy $L \in \mathscr{A}$ with corresponding cash-reserves process $R^{L}\left(R^{L}(0)=x\right)$ and apply Itô's formula to $f\left(t, R^{L}(t)\right)=e^{-r t} V\left(R^{L}(t)\right)$ :

$$
\begin{aligned}
e^{-r t} V\left(R^{L}(t)\right)= & V(x)+\int_{0}^{t} e^{-r s}\left(\mu V^{\prime}\left(R^{L}(s)\right)-r V\left(R^{L}(s)\right)\right) d s \\
& +\frac{1}{2} \int_{0}^{t} e^{-r s} V^{\prime \prime}\left(R^{L}(s)\right) d\left[R^{L}, R^{L}\right]^{c}(s) \\
& +\int_{0}^{t} e^{-r s} a(s) \sigma V^{\prime}\left(R^{L}(s)\right) d W(s)-\int_{0}^{t} e^{-r s} V^{\prime}\left(R^{L}(s)\right) d L(s) \\
& +\sum_{s \in \Gamma} e^{-r s}\left(V\left(R^{L}\left(s_{+}\right)\right)-V\left(R^{L}(s)\right)-V^{\prime}\left(R^{L}(s)\right)\left(R^{L}\left(s_{+}\right)-R^{L}(s)\right)\right),
\end{aligned}
$$

where $\Gamma$ is the set of discontinuities of $L$. Since $L$ is of bounded variation (being and increasing process) we have that

$$
d\left[R^{L}, R^{L}\right]^{c}(s)=\sigma^{2} d s .
$$

Thus, Eq. (20) becomes

$$
\begin{aligned}
e^{-r t} V\left(R^{L}(t)\right)= & V(x)+\int_{0}^{t} e^{-r s} \mathcal{L}^{a}\left(V\left(R^{L}(s)\right)\right) d s \\
& +\int_{0}^{t} e^{-r s} a(s) \sigma V^{\prime}\left(R^{L}(s)\right) d W(s)-\int_{0}^{t} e^{-r s} V^{\prime}\left(R^{L}(s)\right) d L(s) \\
& +\sum_{s \in \Gamma} e^{-r s}\left(V\left(R^{L}\left(s_{+}\right)\right)-V\left(R^{L}(s)\right)-V^{\prime}\left(R^{L}(s)\right)\left(R^{L}\left(s_{+}\right)-R^{L}(s)\right)\right),
\end{aligned}
$$

where $\mathcal{L}^{a}$ is the infinitesimal generator of the diffusion part of $R^{L}$. We now take expectations on both sides and the Itô integral vanishes. On the other hand, by the dynamic programming principle we have

$$
\mathbb{E}\left[e^{-r t} V\left(R^{L}(t)\right)\right] \leq V(x)-\mathbb{E}\left[\int_{0}^{t} e^{-r s} V^{\prime}\left(R^{L}(s)\right) d L(s)\right] .
$$

These two facts yield

$$
\begin{aligned}
0 \geq & \mathbb{E}\left[\int_{0}^{t} e^{-r s}\left(-r V\left(R^{L}(s)\right)+\mu a(s) V^{\prime}\left(R^{L}(s)\right)+\frac{1}{2} \sigma^{2} a^{2}(s) V^{\prime \prime}\left(R^{L}(s)\right)\right) d s\right] \\
& +\mathbb{E}\left[\sum_{s \in \Gamma} e^{-r s}\left(V\left(R^{L}\left(s_{+}\right)\right)-V\left(R^{L}(s)\right)-V^{\prime}\left(R^{L}(s)\right)\left(R^{L}\left(s_{+}\right)-R^{L}(s)\right)\right)\right] .
\end{aligned}
$$

For the set $\Gamma$, by the Mean Value Theorem there exists $r \in\left(R^{L}\left(s_{+}\right), R^{L}(s)\right)$ such that

$$
V\left(R^{L}\left(s_{+}\right)\right)-V\left(R^{L}(s)\right)=V^{\prime}(r)\left(R^{L}\left(s_{+}\right)-R^{L}(s)\right) .
$$

Therefore

$$
\begin{aligned}
& V\left(R^{L}\left(s_{+}\right)\right)-V\left(R^{L}(s)\right)-V^{\prime}\left(R^{L}(s)\right)\left(R^{L}\left(s_{+}\right)-R^{L}(s)\right) \\
& =\left(V^{\prime}\left(r^{*}\right)-V^{\prime}\left(R^{L}(s)\right)\right)\left(R^{L}\left(s_{+}\right)-R^{L}(s)\right)
\end{aligned}
$$


and by concavity of $V$ the right hand side is positive. We have that the second summand of the right-hand side of Expression (22) is positive. This yields

$$
0 \geq \mathbb{E}\left[\int_{0}^{t} e^{-r s}\left(-r V\left(R^{L}(s)\right)+\mu V^{\prime}\left(R^{L}(s)\right)+\frac{1}{2} \sigma^{2} V^{\prime \prime}\left(R^{L}(s)\right)\right) d s\right] .
$$

Next we multiply both sides of the equation above times $1 / t$. Since

$$
\begin{aligned}
& \frac{1}{t} \int_{0}^{t} e^{-r s}\left(-r V\left(R^{L}(s)\right)+\mu V^{\prime}\left(R^{L}(s)\right)+\frac{1}{2} \sigma^{2} V^{\prime \prime}\left(R^{L}(s)\right)\right) d s \\
& \quad \leq \max _{s \in[0, t]} e^{-r s}\left|-r V\left(R^{L}(s)\right)+\mu V^{\prime}\left(R^{L}(s)\right)+\frac{1}{2} \sigma^{2} V^{\prime \prime}\left(R^{L}(s)\right)\right|,
\end{aligned}
$$

we may apply Lebesgue's Dominated Convergence Theorem, and take limit as $t \rightarrow 0$ inside the expectation operator, which results in

$$
0 \geq\left\{\frac{1}{2} \sigma^{2} V^{\prime \prime}(x)+\mu V^{\prime}(x)-r V(x)\right\} .
$$

To prove that one of the inequalities is always tight, we resort again to the dynamic programming principle and write for $t>0$

$$
V(x)=\max _{L \in \mathscr{A}} \mathbb{E}\left[\int_{0}^{t} e^{-r s} d L(s)+e^{-r t} V\left(R^{L}(t)\right)\right] .
$$

Inserting Eq. (21) in the equation above we obtain

$$
\begin{aligned}
0= & \max _{L \in \mathscr{A}}\left\{\mathbb{E}\left[\int_{0}^{t} e^{-r s} \mathcal{L}^{a}\left(V\left(R^{L}(s)\right)\right) d s\right]\right. \\
& +\mathbb{E}\left[\int_{0}^{t} e^{-r s}\left(1-V^{\prime}\left(R^{L}(s)\right)\right) d L(s)\right] \\
& \left.+\mathbb{E}\left[\sum_{s \in \Gamma} e^{-r s}\left(\Delta V\left(R^{L}(s)\right)-V^{\prime}\left(R^{L}(s)\right) \Delta R^{L}(s)\right)\right]\right\} .
\end{aligned}
$$

If we write $\tilde{L}$ and $\tilde{G}$ for the continuous parts of $L$ and $G$ respectively, then Eq. (23) above may be rewritten as

$$
\begin{aligned}
0= & \max _{L \in \mathscr{A}}\left\{\mathbb{E}\left[\int_{0}^{t} e^{-r s} \mathcal{L}^{a}\left(V\left(R^{L}(s)\right)\right) d s\right]\right. \\
& +\mathbb{E}\left[\int_{0}^{t} e^{-r s}\left(1-V^{\prime}\left(R^{L}(s)\right)\right) d \tilde{L}(s)\right] \\
& \left.+\mathbb{E}\left[\sum_{s \in \Gamma_{1}} e^{-r s}\left(\Delta V\left(R^{L}(s)\right)+\Delta L(s)\right)\right]\right\} .
\end{aligned}
$$

Notice that for all $s \in(0, t)$ it holds that, for all $s \in \Gamma$,

$$
\Delta V\left(R^{L}(s)\right)+\Delta L(s)=\int_{R^{L}(s)-\Delta L(s)}^{R^{L}(s)}\left(1-V^{\prime}(x)\right) d x \leq 0
$$

This implies all summands on the right-hand side of Eq. (23) are non positive, which concludes the proof. 
Proof of Theorem 2.4 Consider an arbitrary strategy $L \in \mathscr{A}$, and an initial level of cash reserves $x \geq 0$. Recall that the corresponding cash-reserves process evolves according to the SDE

$$
\left.d R^{L}(t)=\mu d t+\sigma d W(t)\right)-d L(t), R^{L}(0)=x .
$$

Proceeding as in the proof of Theorem 2.3 we use (the generalized) Itô's formula applied to $f(t, x)=e^{-r t} V(x)$ and obtain, after simplifications (recall that $\mathcal{L} V\left(R^{L}(t)\right) \leq 0$ )

$$
\begin{aligned}
e^{-r t} \mathbb{E}\left[V\left(R^{L}(t)\right)\right] \leq & V(x)-\mathbb{E}\left[\int_{0}^{t} e^{-r s} V^{\prime}\left(R^{L}(s)\right) d \tilde{L}(s)\right] \\
& +\mathbb{E}\left[\sum_{s \in \Gamma} e^{-r s}\left(V\left(R^{L}\left(s_{+}\right)\right)-V\left(R^{L}(s)\right)\right)\right],
\end{aligned}
$$

where $\tilde{L}(s):=L(s)-\Delta L(s)$ is the continuous parts of $L$. Let $s \in \Gamma$, then by the Mean Value Theorem and the fact that $1 \leq V^{\prime}\left(R^{L}(s)\right)$, there exists $r \in\left(R^{L}\left(s_{+}\right), R^{L}(s)\right)$ such that

$$
V\left(R^{L}\left(s_{+}\right)\right)-V\left(R^{L}(s)\right)=V^{\prime}(r)\left(R^{L}\left(s_{+}\right)-R^{L}(s)\right) \leq L\left(s_{+}\right)-L(s)=-\Delta L(s) .
$$

Inserting the above expressions into Expression (24) we get

$$
e^{-r t} \mathbb{E}\left[V\left(R^{L}(t)\right)\right] \leq V(x)-\mathbb{E}\left[\int_{0}^{t} e^{-r s} d L(s)\right] .
$$

By continuity $V(x)$ is bounded for $x \in\left[0, x^{*}\right]$ and it grows linearly as $x$ tends to infinity, therefore

$$
\lim _{t \rightarrow \infty} e^{-r t} \mathbb{E}\left[V\left(R^{L}(t)\right)\right]=0 .
$$

This implies that

$$
V(x) \geq \mathbb{E}\left[\int_{0}^{\infty} e^{-r s} d L(s)\right] .
$$

Next consider the strategy $L^{*}$. Since $L^{*}$ is the local time of $R^{*}$ at levels $x^{*}$, we may assume that $x \in\left[0, x^{*}\right]$. Furthermore, $L^{*}$ is a continuous process, and on $\left[0, x^{*}\right]$ it holds that $\mathcal{L}\left(V\left(R^{L^{*}}(s)\right)\right)=0$. Hence, for the strategy $L^{*}$ Itô's formula yields

$$
\begin{aligned}
e^{-r t} V\left(R^{L^{*}}(t)\right)= & V(x)+\int_{0}^{t} e^{-r s} a^{*}(s) \sigma V^{\prime}\left(R^{L^{*}}(s)\right) d W(s) \\
& -\int_{0}^{t} e^{-r s} V^{\prime}\left(R^{L^{*}}(s)\right) d L^{*}(s)
\end{aligned}
$$

The measure $d L^{*}(s)$ is supported on $\left\{R^{L^{*}}(s)=x^{*}\right\}$ and $V^{\prime}\left(x^{*}\right)=1$, therefore, taking expectations, Eq. (26) may be rewritten as

$$
e^{-r t} \mathbb{E}\left[V_{\beta}\left(R^{L^{*}}(t)\right)\right]=V_{\beta}(x)-\mathbb{E}\left[\int_{0}^{t} e^{-r s} d L^{*}(s)\right] .
$$

Letting $t \rightarrow \infty$ we have

$$
V(x)=\mathbb{E}\left[\int_{0}^{\infty} e^{-r s} d L^{*}(s)\right]
$$


which is equivalent to $V(x)=V^{L^{*}}(x)$. Form Eq. (25) we have that for any $L \in \mathscr{A}$, $V(x) \geq V^{L}(x)$. Since $L^{*} \in \mathscr{A}$ Eq. (27) yields

$$
V(x)=\sup _{L \in \mathscr{A}} V^{L}(x),
$$

which concludes the proof.

Proof of Proposition 3.2 Consider an initial level of cash reserves $x$, an admissible triple $(a, L, J)$ and the corresponding value $V(x)$. We may assume without loss of generality that $R\left(\tau_{1}\right)>0$. Let us define the auxiliary processes

$$
\tilde{J}(t):=\sum_{i=2}^{\infty} x_{i} \mathbb{1}_{\left\{t \geq \tau_{i}\right\}}
$$

and the corresponding $\tilde{R}(t)$, together with the stopping time $\tau:=\inf \{t>0 \mid \tilde{R}(t) \leq 0\}$. By construction $\tau>\tau_{1}$. If we define $\bar{J}(t):=\tilde{J}(t)+x_{1} \mathbb{1}_{\{t \geq \tau\}}$ we have that the triple $(a, L, \bar{J})$ is admissible and the corresponding value $U(x)$ satisfies $U(x)>V(x)$. This last assertion follows from the facts that $-e^{-r \tau}\left(x_{1}+\kappa\right)<e^{-r \tau_{1}}\left(x_{1}+\kappa\right)$, and that for any $t \geq \tau_{2}$ it holds that $R(t)=\tilde{R}(t)$. It is then optimal to postpone the first time of issuance to the first time the cash-reserves process hits zero, and an inductive argument implies that such is the case for all re-injection times. Furthermore, the stationarity of our model also implies that there exists $\hat{x} \geq 0$ such that $x_{i} \equiv \hat{x}$ for all $i \geq 1$.

Proof of Lemma 4.3 Let $V$ be a solution to

$$
-\rho V(x)+r x V^{\prime}(x)-\frac{1}{2}\left(\frac{\mu-r}{\sigma}\right)^{2} \frac{\left(V^{\prime}(x)\right)^{2}}{V^{\prime \prime}(x)}=0, \quad V(0)=0
$$

and define

$$
u(x):=x \frac{V^{\prime}(x)}{V(x)}
$$

We have that

$$
V^{\prime}(x)=\frac{u(x) V(x)}{x},
$$

therefore

$$
V^{\prime \prime}(x)=\frac{x u^{\prime}(x)-u(x)}{x^{2}} V(x)+\frac{u(x) V^{\prime}(x)}{x}=\frac{x u^{\prime}(x)-u(x)+u^{2}(x)}{x^{2}} V(x) .
$$

From the above expression we get

$$
\frac{\left(V^{\prime}(x)\right)^{2}}{V^{\prime \prime}(x)}=\frac{u(x)^{2}}{x u^{\prime}(x)-u(x)+u^{2}(x)} V(x)
$$

If we divide Eq. (28) by $\mathrm{V}(\mathrm{x})$, we obtain

$$
\rho=r u(x)-\frac{\varsigma u^{2}(x)}{x u^{\prime}(x)+u^{2}(x)-u(x)},
$$

where $\zeta:=\frac{1}{2}\left(\frac{\mu-r}{\sigma}\right)^{2}$. This is equivalent to

$$
x u^{\prime}(x)(r u(x)-\rho)=-r u(x)(u(x)-\alpha)(u(x)-\tilde{\alpha}),
$$


where that $0<\alpha<1<\rho / r<\tilde{\alpha})$ are the roots of

$$
f(x):=r x^{2}-(r+\rho+\varsigma) x+\rho .
$$

By separating variables and integrating, we can see that the solutions to Eq. (29) are all such that

$$
|u(x)-\alpha|^{r_{1}}|u(x)-\tilde{\alpha}|^{r_{2}}=\lambda \frac{u(x)}{x},
$$

where $r_{1}:=(\tilde{\alpha}-1) /(\tilde{\alpha}-\alpha), r_{2}:=(1-\alpha) /(\tilde{\alpha}-\alpha)$ and $\lambda$ is determined by the initial condition $V(0)=0$. The latter implies that

$$
\lim _{x \rightarrow 0} \frac{u(x)}{x}=\lim _{x \rightarrow 0} \frac{V^{\prime}(x)}{V(x)}=\infty .
$$

Equations (29) and (30), together with the concavity of $V$, imply that $u(x) \leq \rho / r$. Notice that, as a consequence, the left-hand side of Eq. (31) is then bounded (since $r_{1}, r_{2}>0$ ). Therefore it must hold that $\lambda=0$, which yields $u(x) \equiv \alpha$ or $\tilde{\alpha}$. The latter is ruled out, since $\tilde{\alpha}>\rho / r$, which would violate the concavity of $V$. Thus $u(x) \equiv \alpha$ is the only possibility and we have

$$
V(x)=K x^{\alpha},
$$

where $K$ is a positive constant.

Proof of Lemma 4.5 Let us define $\varsigma:=\frac{1}{2}\left(\frac{\mu-r}{\sigma}\right)^{2}$ and recall that $\alpha$ is the smallest root of

$$
f(x):=r x^{2}-(r+\rho+\varsigma) x+\rho .
$$

Let $\beta$ be the largest root of $f(x)=0$. Proving that $k<1$ is equivalent to showing that $\alpha>1-\frac{\mu-r}{\sigma^{2}}$ Since $f(x)>0$ for $x \in(-\infty, \alpha) \cup(\beta, \infty)$, if we showed that $\beta>1-\frac{\mu-r}{\sigma^{2}}$ then the desired result would follow from showing that

$$
f\left(1-\frac{\mu-r}{\sigma^{2}}\right)>0 .
$$

Indeed, we have that the condition $\beta>1-\frac{\mu-r}{\sigma^{2}}$ is equivalent to

$$
\sqrt{(r+\rho+\varsigma)^{2}-4 r \rho}>r-\rho-\varsigma\left(1+\frac{4 r}{\mu-r}\right),
$$

which trivially holds, since the right-hand side of the expression above is negative from the assumption that $\rho>r$. Next we compute

$$
f\left(1-\frac{\mu-r}{\sigma^{2}}\right)=\frac{\mu-r}{2 \sigma^{2}}\left(2 \rho-\mu-r+2 \varsigma+\frac{2 r}{\sigma^{2}}(\mu-r)\right),
$$

which is greater than zero, given the assumption that $\rho>\mu>r$.

Proof of Lemma 4.6 Observe that the boundary-value problem in hand can be rewritten, for $u>0$ as

$$
\frac{2 \rho}{\sigma^{2}(x+D)^{2}} V_{u}(x)=\frac{2(\mu x+(\mu-r) D)}{\sigma^{2}(x+D)^{2}} V_{u}^{\prime}(x)+V_{u}^{\prime \prime}(x), \quad V_{u}^{\prime}(u)=1, V_{u}^{\prime \prime}(u)=0 .
$$

The mappings

$$
x \mapsto \frac{2 \rho}{\sigma^{2}(x+D)^{2}} \quad \text { and } \quad x \mapsto \frac{2(\mu x+(\mu-r) D)}{\sigma^{2}(x+D)^{2}}
$$


are continuous on $[0, \infty)$. Furthermore, their derivatives on $(0, \infty)$ are bounded, hence they are Lipschitz. The existence and uniqueness of a solution to the boundary-value problem follows then directly from the Cauchy-Lipschitz Theorem.

Proof of Theorem 4.7 Observe that from Lemma 4.6 we immediately get that, for any $x_{1}>$ $x_{0}^{*}$ the linear extension of the function $V_{x_{1}}:\left(x_{0}^{*}, x_{1}\right) \rightarrow \mathbb{R}$ to $\left[x_{1}, \infty\right)$ via the mapping $x \mapsto\left(x-x_{1}\right)+V_{x_{1}}\left(x_{1}\right)$ yields a $\mathcal{C}^{2}$ function on $\left[x_{0}^{*}, \infty\right)$. We are left with the task of showing that the pair $\left(K, x_{1}\right)$ may be chosen in such a way that

$$
K\left(x_{0}^{*}\right)^{\alpha}=V_{x_{1}}\left(x_{0}^{*}\right) \text { and } \alpha K\left(x_{0}^{*}\right)^{\alpha-1}=V_{x_{1}}^{\prime}\left(x_{0}^{*}\right) .
$$

We obtain immediately that $K=V_{x_{1}}\left(x_{0}^{*}\right) \cdot\left(x_{0}^{*}\right)^{-\alpha}$, which leaves us with the equation

$$
V_{x_{1}}^{\prime}\left(x_{0}^{*}\right)=\alpha \frac{V_{x_{1}}\left(x_{0}^{*}\right)}{x_{0}^{*}} .
$$

To finalize the proof we must show that Eq. (32) admits a unique solution $x_{1}^{*}>x_{0}^{*}$. To this end we require the following auxiliary result: For all $x \leq u$, the mapping $u \mapsto V_{u}(x)$ is decreasing, and the mapping $u \mapsto V_{u}^{\prime}(x)$ is increasing. Consider $u<v$ and for $x \leq u$ Let us define $g(x):=V_{u}(x)-V_{v}(x)$. It follows from the facts that $V_{v}(v)<(v-u)+V_{u}(u)$ and $V_{v}^{\prime} \geq 1$ that $g(u)>0$ and $g^{\prime}(u)<0$. By concavity of $V_{v}$ we also have $g^{\prime \prime}(u) \geq 0$. Next we wish to show that $g^{\prime}(x)<0$ for all $x<u$. Assume this were not the case and let $y<u$ be the largest solution to $g^{\prime}(y)=0$. Then for $x \in(y, u)$ we have $g^{\prime}(x)<0$, which implies $g(y)>g(u)>0$ and $g^{\prime \prime}(y)<0$. However, from the differential equation

$$
\rho V(x)=(\mu x+(\mu-r) D) V^{\prime}(x)+\frac{\sigma^{2}}{2}(x+D)^{2} V^{\prime \prime}(x)
$$

we obtain that $g(y)=\frac{\sigma^{2}}{2}(y+D)^{2} g^{\prime \prime}(y)<0$. This is a contradiction, which implies that that $g^{\prime}(x)<0$ for all $x<u$; thus $u \mapsto V_{u}^{\prime}(x)$ is increasing. Since $g(u)>0$ we conclude that $g(x)>0$ for all $x<u$, thus $u \mapsto V_{u}(x)$ is decreasing. Furthermore, since $V_{x_{1}}^{\prime} \geq 1$, we have from the Mean Value theorem for $x_{1}>x_{0}^{*}$

$$
V_{x_{1}}\left(x_{0}^{*}\right) \leq V_{x_{1}}\left(x_{1}\right)-\left(x_{1}-x_{0}^{*}\right)=\frac{\mu x_{1}+(\mu-r) D}{\rho}-\left(x_{1}-x_{0}^{*}\right) .
$$

Given the assumption that $\mu<\rho$, we have that $\lim _{x_{1} \rightarrow \infty} V_{x_{1}}\left(x_{0}^{*}\right)=-\infty$. Since $u \mapsto V_{u}^{\prime}(x)$ is an increasing mapping, all we have left to prove is that

$$
V_{x_{0}^{*}}^{\prime}\left(x_{0}^{*}\right)<\alpha \frac{V_{x_{0}^{*}}\left(x_{0}^{*}\right)}{x_{0}^{*}} .
$$

Using the fact that $V_{x_{0}^{*}}^{\prime}\left(x_{0}^{*}\right)=1$, this condition may be rewritten as

$$
0<\alpha\left(\mu x_{1}+(\mu-r) \frac{D}{x_{0}^{*}}\right)-\rho .
$$

from the expression $x_{0}^{*}=\frac{k D}{1-k}$ and the fact that $\frac{1}{k}=\frac{\sigma^{2}(1-\alpha)}{\mu-r}$ we have the above expression is equivalent, after simplifications, to

$$
0<\frac{1}{1-\alpha}(2 \alpha \varsigma+\alpha(1-\alpha) r-\rho+\alpha \rho) .
$$


Recall, however, that by definition $\alpha$ satisfies $r \alpha^{2}+\rho=(r+\rho+\varsigma) \alpha$; thus we are required to show that

$$
0<\frac{1}{1-\alpha}(2 \alpha \varsigma+\alpha(1-\alpha) r-\rho+\alpha \rho)=\frac{1}{1-\alpha} \frac{\alpha \varsigma}{1-\alpha},
$$

which trivially holds, since $\alpha \in(0,1)$ and $\varsigma>0$. This finalizes the proof.

Proof of Propositions 4.8 and 4.9 Recall that $k=\frac{\sigma^{2}}{\mu-r}(1-\alpha)$. Since $(r+\rho+\varsigma)^{2}-4 r \rho=$ $(\rho-r+\varsigma)^{2}+4 r \varsigma$, we may write

$$
1-\alpha=\frac{r-\rho-t+\sqrt{(\rho-r+\varsigma)^{2}+4 r \varsigma}}{2 r},
$$

where $\varsigma$ is as in the proof of Lemma 4.5. Multiplying both numerator and denominator of $1-\alpha$ times $-(r-\rho-\varsigma)+\sqrt{(\rho-r+\varsigma)^{2}+4 r \varsigma}$ an simplifying we obtain

$$
1-\alpha=\frac{2 \varsigma}{\rho-r+\varsigma+\sqrt{(\rho-r+\varsigma)^{2}+4 r \varsigma}}=\frac{2}{\frac{\rho-r}{\varsigma}+1+\sqrt{\left(\frac{\rho-r}{\varsigma}+1\right)^{2}+4 \frac{r}{\varsigma}}} .
$$

From Eq. 33 we conclude that $1-\alpha$ and, therefore $k$, is decreasing in $r h o-r$ and increasing in $\varsigma$. Given that the latter is decreasing in $\sigma$ and increasing in $\mu-r$, Proposition 4.9 is proved. Using Eq. 33 we have that

$$
k=\frac{\mu-r}{\rho-r+\varsigma+\sqrt{(\rho-r+\varsigma)^{2}+4 r \varsigma}} .
$$

We immediately observe that $k$ is decreasing in $\rho-r$. Furthermore, since $\varsigma$ is decreasing in $\sigma$, then $k$ is increasing in the volatility of assets. To show that $k$ is single-peaked in $\mu-r$, observe that

$$
\frac{1}{k}=\frac{\rho-r}{\mu-r}+\frac{\mu-r}{2 \sigma^{2}}+\sqrt{\frac{2 r}{\sigma^{2}}+\left(\frac{\rho-r}{\mu-r}+\frac{\mu-r}{2 \sigma^{2}}\right)^{2}}
$$

is an increasing function of

$$
v(\mu-r):=\frac{\rho-r}{\mu-r}+\frac{\mu-r}{2 \sigma^{2}} .
$$

Notice that $\lim _{x \rightarrow 0} v(x)=\lim _{x \rightarrow \infty} v(x)=\infty$ and $v^{\prime}(x)=0$ has a unique positive solution $x=\sigma \sqrt{2} \sqrt{\rho-r}$. In other words, $v$ is itself an U-shaped function of $\mu-r$, which implies that $k$ is single peaked in $\mu-r$. This concludes the proof.

\section{References}

1. Akyildirim, E., Güney, I.E., Rochet, J.-C., Soner, H.M.: Optimal dividend policy with random interest rates. J. Math. Econ. 51, 93-101 (2014)

2. Barth, A., Moreno-Bromberg, S.: Optimal liquidity and risk management with costly refinancing opportunities. Insur.: Math. Econ. 57, 31-45 (2014)

3. Barth, A., Moreno-Bromberg, S., Reichmann, O.: A non-stationary model of optimal dividend distribution in a stochastic interest-rate setting. Tech. report, Swiss Finance Institute, (2014)

4. Bass, R.F., Hsu, P.: The semimartingale structure of reflecting Brownian motion. Proc. Am. Math. Soc. 108(4), 1007-1010 (1990) 
5. Brunnermeier, M., Sannikov, Y.: A macroeconomic model with a financial sector. Am. Econ. Rev. 104(2), 379-421 (2014)

6. Décamps, J.-P., Mariotti, T., Rochet, J.-C., Villeneuve, S.: Free cash flow, issuance costs, and stock prices. J. Financ. 66, 1501-1544 (2011)

7. Dumas, B.: Super contact and related optimality conditions. J. Econ. Dyn. Control 15(4), 675-685 (1991)

8. Franks, J.R., Sanzhar, S.V.: Evidence on debt overhang from distressed equity issues, Tech. report, Working Paper, London Business School (2006)

9. Hoejgaard, B., Taksar, M.: Controlling risk exposure and dividends pay-out schemes: insurance company example. Math. Financ. 9, 153-182 (1998)

10. Hugonnier, J., Morellec, E.: Bank capital, liquid reserves, and insolvency risk. Tech. report, Swiss Finance Institute and EPFL (2014)

11. Isohätälä, J., Milne, A., Robertson, D.: A model of investment subject to financing constraints. Loughborough University and University of Cambridge, Tech. report (2012)

12. Jeanblanc-Picqué, M., Shiryaev, A.N.: Optimization of the flow of dividends. Uspekhi Mat. Nauk 2(203), 25-46 (1995)

13. Jiang, Z., Pistorius, M.: Optimal dividend distribution under Markov regime switching. Financ. Stoch. 16(3), 449-476 (2012)

14. Karatzas, I., Shreve, S.E.: Brownian motion and stochastic calculus, graduate texts in mathematics. Springer, Berlin (1991)

15. Klimenko, N., Moreno-Bromberg, S.: The shadow cost of short-term debt and corporate policies. Deparment of Banking and Finance, University of Zurich, Tech. report (2014)

16. Kruk, L., Lehoczky, J., Ramanan, K., Shreve, S.E.: An explicit formula for the Skorokhod map on [0, $a]$. Ann. Probab. 35(5), 1740-1768 (2007)

17. Merton, R.C.: Lifetime portfolio selection under uncertainty: the continuous-time case. Rev. Econ. Stat. 51(3), 247-257 (1969)

18. Milne, A., Robertson, D.: Firm behavior under the threat of liquidation. J. Econ. Dyn. Control 20, 1427-1449 (1996)

19. Pötzelberger, K., Wang, L.: Boundary crossing probability for brownian motion and general boundaries. J. Appl. Probab. 34(1), 54-65 (1997)

20. Radner, R., Shepp, L.: Risk vs. profit potential: a model for corporate strategy. J. Econ. Dyn. Control. 1371-1393 (1996)

21. Rochet, J.-C., Villeneuve, S.: Corporate portfolio management. Ann. Financ. 1(3), 225-243 (2005)

22. Rochet, J.-C., Villeneuve, S.: Liquidity management and corporate demand for hedging and insurance. J. Financ. Intermed. 1(20), 303-323 (2011)

23. Ross, S.A., Westerfield, R.W., Jordan, B.D.: Fundamentals of corporate finance. McGraw-Hill/Irwin, New York, NY (2008)

24. Skorokhod, A.V.: Stochastic equations for diffusion processes in a bounded region. Theory Probab. Appl. 6(3), 264-274 (1961) 